\title{
Understanding Virtual Actors
}

\author{
Rogério E. da Silva \\ Dept. Information Systems, \\ University of Minho, Portugal and \\ State University of Santa Catarina, Brazil \\ rsilva@joinville.udesc.br
}

\author{
Ido. A. Iurgel \\ Computer Graphics Center and \\ Dept. Information Systems, \\ University of Minho, Portugal \\ ido.iurgel@dsi.uminho.pt \\ Nelson Zagalo \\ Dept. Communication Sciences, \\ University of Minho, Portugal \\ nzagalo@ics.uminho.pt
}

\author{
Manuel F. dos Santos, Pedro Branco \\ Dept. Information Systems, \\ University of Minho, Portugal \\ $\{m f s, p b r a n c o\} @ d s i . u m i n h o . p t$
}

\begin{abstract}
Autonomous Digital Actors represent the next step in authoring movies with believable characters, in a way that will allow them to be trained for acting specific roles in a story, suggesting appropriate behaviors during their performance. This article presents an overview of the art of acting and directing and how these concepts were used to elaborate a Virtual Actor metaphor. Also, we present an agent architecture for describing and implementing the virtual actors' acting knowledge base.
\end{abstract}

Keywords-Virtual Actors; Autonomous Digital Actors; Authoring Believable Characters; Computer Animation

\section{INTRODUCTION}

Since Disney first introduced his 'Principles of Animation', animators have searched for ways to deliver more and more compelling believable characters. During the past decades, animation has evolved into computer animation and, with the aid of computers, characters could finally be synthetically generated. Computer models emerged, relying on several different sciences and approaches to increase believability.

Technology has achieved an incredible realistic levels in terms of appearence but regarding character's agency, they need humans to make all kinds of decisions for them. For instance, the way they should perform while interpreting a role in a story. The next evolutionary step for computer animation will most certainly consist of behavior simulation; this should allow characters to act by themselves, delivering autonomous performances out of stories.

In fact, Perlin \& Seidman has already foreseen that " $3 D$ animation and gaming industry will soon be shifting to a new way to create and animate $3 D$ characters, and that rather than being required to animate a character separately for each motion sequence, animators will be able to interact with software authoring tools that will let them train an Autonomous Digital Actor (ADA) how to employ various styles of movement, body language, techniques for conveying specific emotions, best acting choices, and other general performance skills" [1].
This work is particularly interested in the application of believable characters to the development of virtual actors ${ }^{1}$. Our understanding is that a virtual actor is an analogy to a real actor, which autonomously, and by its independent interpretation of the situation, can perform its role according to a given script, as part of a story [2], [3].

If one wants to create autonomous characters acting analogously to real actors, the first step should be to understand the art of acting and how actors learn their art, and how they prepare for an acting role. Another important aspect that needs to be learned is how directors and actors work together. Their relations can tell us a lot about how authoring virtual actors could be made intuitive.

The aim of this article is to present and discuss several studies made over the past few years regarding the art of acting and directing, character animation and the authoring process. Departing from these studies, we developed a virtual acting framework composed by three main modules: script annotation, behavior suggestion and animation scheduling.

This article is divided into four sections where: section I is this introduction, section II presents a literature review about real acting and directing, and related works. Then, section III discusses our ideas regarding the development of a virtual actors framework. Finally, section IV presents some conclusions.

\section{LiterATURE REVIEW}

\section{A. The Art of Acting}

Acting literally means to perform an action. From dramatic arts, as in theater, filmmaking or television, acting is understood as interpreting a part or a role in a story. There are basically two types of actors: theater actors and screen actors, and they differ significantly in the way they view their performances.

\footnotetext{
${ }^{1}$ We use the terms Virtual Actor and Autonomous Digital Actors interchangeably.
} 
On the one hand, theater actors have a tendency for exaggeration and for stylization, for what could be considered unnatural. Also, theater actors deliver life performances, which means that the audience is observing the action while it is being performed. Screen actors, on the other hand, deliver recorded performances in front of a camera in a set, that are later edited into a final piece, that is finally viewed by the audience.

\section{1) Acting Schools: Constantin Stanislavski}

Constantin Stanislavski, a Russian actor and director, was a legendary acting teacher whose lessons are still today being followed by acting students worldwide. He developed a method for acting based on the following insights [4], [5]:

- Imagination and Emotions: imagination is a powerful source of emotions. A performance without emotions makes it look artificial.

- Concentration and Relaxation: an actor should focus his/her attention exclusively on the performance and, an actor should learn how to relax his/her muscles to foster believable movements while performing.

- Objectives, obstacles and actions: a performance should be explained as objectives (what motivates the characters to act?), obstacles (what stops him/her from getting his/her objectives done?) and actions (what does he to achieve his/her goals?). Stanislavski warns to be careful not try to detail too much the performance. Only the most central elements should be described (the general idea).

- Emotion Memory: an actor should recall his/her own previous experiences and the emotions involved in those (not trying to mimic others), which should allow him to retrieve specific behaviors if needed.

- Sense of Truth: truth on stage is anything that the audience can rely on, which is not the same as to replicate the real world. An actor should start deciding how to perform 'from inside' (role character's point of view) and avoid, by all means, overacting (including too much acting details).

- Adaptation: being able to adjust the actor's performance regarding changes of other actors or the surrounding environment.

- Unbroken Line: is a line of action that relates all moments throughout the entire play.

\section{The Method}

Lee Strasberg, a former student of Stanislavski, and cofounder of Actor's Studio (one of the most prestigious American acting schools), developed "The Method", which is (according to Strasberg himself) a continuation of the Stanislavski method. The Method relies on the following fundaments:

- Relaxation and Concentration: following Stanislavski, Strasberg also stresses the importance of the preparation before acting including being relaxed and focused for believability.
- Sense Memory: an actor should recall objects and sensory experiences, for instance, how were the sensations when drinking a cup of coffee (considering all five senses).

- Emotion Memory: unlike sense memory, emotion memory tries to recall emotional experiences from the actor's past, instead of trying to force a reaction or emotion.

- Characterization: an actor should know how to build physically and psychologically a character, using both the sense and emotion memory for that.

- Character's Drives: when preparing for a role, method actors should fully understand their characters in order to perform them believably. Therefore, they are guided to 'answer' questions like "who is the character"? "what does he want"? "why does he want it"? "how is he planning to achieve his/her goals"?

\section{Other Schools}

Appart from Stanislavski and Strasberg methods, other acting teachers (often followers of these two) have developed or adapted their own acting methods. Two well known schools are the Meisner method and the Adler method [5].

- Sanford Meisner insights:

- An actor should perform as if he were living in the real world.

- A performance should be understood as a series of independent interconnected moments.

- The power of imagination is a much more reliable source than sense memory and should be used instead.

- Stella Adler maintains that:

- Every performed action must have a justification, otherwise it should be avoided.

- Performances are representations of observations of the real world.

- To fully understand his/her character, an actor should exhaustively study the script.

- To give 'size' to a character, an actor should rehearse his/her body and voice.

\section{B. The Art of Directing}

Another important issue involved in the creation of dramaturgical pieces is relating to directing. The director is the person responsible for 'guiding' actors' performances toward a common objective. According to Weston [6], good direction is a playable direction, the one that leads to behavior in the actor. She presented two types of directing: the first result-oriented directing, she considered inappropriate due to its "unplayability"; Weston then suggests some "quick fixes' to solve this problem.

- Result-Oriented Directing: Weston defined resultoriented directing as "an attempt to shape the actor's performance by describing the result you are after, i.e., 
how you want it to end up looking or sounding". She illustrated at least ten situations that lead to resultoriented directing, so they could be avoided:

1) Telling actors what effect the director wants as in "can you make it more quirky?".

2) Asking general or vague instructions like "can you give it more energy?".

3) Giving the actor a line reading, which means telling him the expected way of acting (like voice inflection, for instance).

4) Telling the actor what feeling the character should be having or any other mental state (e.g., "I think he is disappointed").

5) An extension of the previous statement is when the director tries to tell the actor what reaction to have as in "when she tells you she doesn't have the money you get angry"

6) Even worse happens when the director gives a fully loaded emotional map to the actor: "when the scene starts he is worried because she is late. He is relieved when she arrives, but then disappointed because she hasn't got the money, and then he becomes suspicious that she might be holding out on him".

7) Telling about how the director understands the character is unproductive.

8) Directors quite often gives confusing/unplayable directions like "can you play him aggressive, but pleasant?"

9) Negative judgments: it is not up to the director (or the actors for that matter), the responsible for judging the character and its actions as in "he is a punk".

10) The director should not be the one deciding the character's attitude like "it has a hostile attitude toward his father".

- Quick Fixes: Good direction should be: active, objective, sensory, dynamic and kinetic. These quick fixes represent five tools that aim at responding each of these properties respectively:

- Verbs: one common mistake directors do is to suggest actions using adjectives (e.g. "be defensive") when they should use verbs instead, because verbs denote experiences instead of conclusions of experiences (results). But not every verb is appropriate, only transitive verbs that take an object, because they have an emotional and a physical component. A suggested list of such verbs can be found at [6].

- Facts: there are two kinds of facts: factual backstory and imaginative backstory. Facts are always self-explanatory and should never need further explanations.

- Images: images actually relates to what we per- ceive of the world using all our five senses. Images can easily trigger emotions than explanations.

- Events: events are the central idea of every scene. They are used to indicate to actors what is the story being told or the theme of the movie being made. Such an understanding is vital for actors to be able to imagine which are (and why) the appropriate reactions while performing the scene.

- Physical Tasks: sometimes actors can enter in a state called 'self-consciousness', which is thinking about their own performance. Giving them some extra physical task (like try to open an stuck door while speaking his lines) can be a very efficient way to maintain actors away from selfconsciousness and delivering compelling performances.

\section{Script Analysis}

There is a consensus among acting teachers that an actor should study his/her role character in depth to prepare himself for his/her part. These items summarize important aspects of studying a script [7], [8].

- Action Analysis: it is the first analysis of the script. It aims at a less complex analysis than formalist analysis (the one resulted by the sum of all the next aspects of script analysis), and it is responsible for detecting from the text the following elements:

- Sequence of External Events: events that change characters.

- Review of the Facts: describe who are the main characters in each event, what they are doing, where the action takes place, why it happens, when it happens.

- Seed: describe a moral commandment that has been violated, and then justified the action.

- Sequence of Internal Events: describe the connections between the seed and the external events.

- Three Major Climaxes: define the three major turning points of the story (the beginning, middle and end).

- Theme: what was the main character's response to the seed?

- Super-Objective: is the goal that the main character is striving to achieve.

- Through-Action: state the main conflict of the play in one concise sentence.

- Counter Through-Action: what interfere with the through-action of the main character.

- Given Circumstances: describe, according to Stanislavski, the unique combinations of past and present situations that mark the beginning of the play. In [7], it is described a series of given circumstances that occur in the present as follows (next topic 
"Background Story" describes the given circumstances that occur in the past):

- Time: describe when the play takes place, how the passage of time is perceived by the audience.

- Place: describe where the story happens and its properties.

- Society: describe the relationships between people living in 'place'.

- Economics: describe, in general terms, how is the economic system of the play, how do the 'society' lives in this system? How money influence their lives?

- Politics and Law: what is the government system? and how this influence peoples lives?

- Learning and the Arts: what is the general level of culture and artistic taste of the characters?

- Spirituality: describe characters' spiritual and religious beliefs.

- The World of the Play: means how do all previous given circumstances work together? what is the 'the world' that character live in?

- After Action Analysis: how do the 'Seed/Theme' relate with these given circumstances?

- Background Story: indicates everything that happened before the beginning of the play.

- External and Internal Actions: external actions represent all the entrances (the moments a character starts his part in a dialog) and exits (the moment he stops his participation). Internal actions describe assertions about people, places, things and events during the action.

- Progressions and Structure: progressions are subdivisions of the play into units (or beats), scenes and acts. Structure defines the motivating force for the story, main conflicts, the more and the less intense emotional points.

- Character: is defined in terms of:

- Objectives: what is his super-objective? and his minor objectives for each scene, unit and beat?

- Conflicts: describes what are the main characters' conflicts (in terms of role and objectives)? when they occur? what complication can happen?

- Will Power: how does the character work to carry out his/her objectives? steady? vacillate?

- Values: what does each character stands for and against? What he believes to be right and wrong? Good and bad?

- Personality Traits: describe each character in terms of: energy level, consistency of behavior, impulses and inhibitions.

- Complexity: how self-aware is the character? Who are the type, intermediate and complex characters?

- Relationships: what are the character's primary and secondary relationships?
- Idea: is represented by:

- Words: describe the meanings of the play in terms of title, speeches, illustrations, allusions, etc.

- Characters: is there a narrator or chorus? which other types of characters there are in the story: skeptical, confidant, and so on.

- Plot: are there any repetitive situation? intellectual conflicts? symbolism?

- Statement of the Main Idea: explains the main idea of the story in a single concise sentence.

- Mise-en-scene: how the main idea influences and contributes to the mise-en-scene?

- Dialogue: is described as:

- Words: what kind of words are used in the dialogs? concrete? abstract? formal? informal? professional jargon? slangs?

- Sentences: what is the average length of the sentences? what types of sentences are? any special kind?

- Speeches: For what purposes do the speeches were written? How do they were being connected? By words? By thoughts? By meanings?

- Special Qualities: is the dialog written in verse? or prose?

- Theatricality: how does it expresses external and internal actions? how emotional it is? is there any subtext? how?

- Tempo, Rhythm and Mood: considering the tempo, how often information is presented about plot or about characters? and about rhythm, how do the emotional tensions work on each scene, act or the entire play? how this affect each character? And finally, considering the mood, are there any atmospheric feelings with a given circumstance? or character? or idea? what controls the atmosphere of the world of the play?

\section{Embodied Conversational Agents}

One of the most basic skills that any actor should master while performing a role is to engage in dialogs with other actors. Learning how people behave during a conversation is vital to actors delivering a believable performance. This is no different concerning virtual actors. Being able of believably engaging dialogs it is the first skill they should learn.

Embodied Conversational Agents (or ECA) [9] are characters capable of engaging in a face-to-face conversation autonomously deciding for proper behaviors considering four main functions: emotional, personality, performative and conversational. Next sections briefly discuss each of these functions.

1) Emotional Function: In [10], emotion is defined as "short-term variation in internal mental states" (either physical like fear or cognitive like jealousy).

It is important for virtual characters to understand situations that trigger emotions and proper ways of displaying 
such states. There are several computational models one could rely on, being the OCC model [11] one of the most popular.

Ball and Breese presented an emotional model that considers a two dimensional system: valence indicates the positive and negative dimension of a feeling while the arousal indicates the degree of intensity of the emotional response. Valence has been discretized as negative, neutral and positive. Arousal as excited, neutral and calm. The combination of both variables that can be labeled as an emotion.

2) Personality Function: Similarly as occurs with emotions, psychologists do not agree on a single definition for personality. Consequently, several theories (and models) resulted from this ${ }^{2}$.

For instance, [10] stress that personality is "a permanent (or very slowly changing) pattern of thoughts, emotion, and behavior associated with an individual". They use a two dimensional model (similar to their emotion model) based on the variables: dominance (individual's relative disposition towards controlling others) and friendliness (tendency to be warm or sympathetic).

One of the most popular personality models (the OCEAN model) describe personalities by five traits: openness, conscientiousness, extraversion, agreeableness and neuroticism [12].

3) Performative Function: People can communicate verbally (words and intonation), non-verbally (gestures, gaze, posture) and para-verbally (sounds). Usually, we synchronize several of these channels with the utterance. This is made for several reasons: to emphasize something, to explain something, to illustrate something, etc.

Performative functions aim at deciding how each behavior should be used during a conversation and, more important, how to synchronize different channels to produce more appealing results in communicating with others.

In [13], for instance, it is discussed the importance of such performative skills in conversational agents. The authors also proposed a way for creating artificial agents capable of displaying its communicative intentions through facial expressions.

4) Conversational Function: Naturally, to engage in a conversation, a person must be able to understand what it is being said. Speech analysis is responsible for, given a speech, producing abstract representations of words, sentences, dialog structure, semantics in such a way that allows characters to work with these representations to infer proper behaviors.

Torres et al [14] argue that when people engage in a conversation they take turns speaking which "almost always begin and end smoothly, with short lapses of time between

\footnotetext{
${ }^{2}$ Personality theories list: http://webspace.ship.edu/cgboer/perscontents. html
}

them". So, by simply understanding when each take begins and ends, a character can infer coherent behaviors in these moments; however, for everything in between he can not. So, a more detailed representation is needed: a discourse structure representation.

The authors work with a representation that subdivides utterances into theme (the part of the utterance that links it with the previous one) and rheme (the part that introduces new content to the conversation). In [15], [14], [9] this rheme/theme representation is discussed in more details. Once one has a hierarchical structure of the discourse, it is possible to infer behaviors in a much more detailed manner (see the BEAT system in section II-E1 for an example of a conversational function).

Other possibilities for representing discourse structure are: syntax structure ${ }^{3}$ and conceptual structure [16].

\section{E. Related Works}

From the past few years, several researchers have published works that in a sense, have contributed towards the development of virtual actors. Next sections present some of these works, discussing strengths and weaknesses relatively to the development of virtual actors.

1) BEAT: (or Behavior Expression Animation Toolkit) "allows one to animate a human-like body using just text as input" [17].

The system is composed by three main modules:

1) the Language Tagging module is responsible for "annotating the input text with linguistic and contextual information". This is made by converting the text into a tree representation of the most important units (utterance, clause, theme/rheme, action and/or object).

2) the Behavior Generation module is responsible for generating appropriate behaviors after analyzing a previously annotated sentence by the language tagging module. This behavior generation is divided in two sub-modules:

- Behavior Suggestion: takes the resulting tree from tagging a sentence and produces a list of possible (coherent) behaviors for each node in it.

- Behavior Selection: is composed by a series of filter that try to remove from the behavior's list of each node, those have not been considered 'playable' like, for instance, due to two different behaviors that try to manipulate the same DOF at the same time.

3) finally, the Behavior Scheduling module takes the resulting tree and convert each of its nodes into abstract animation commands for the characters. Also, it is responsible for translating such abstract commands into intelligible commands for a selected animation tool.

${ }^{3}$ http://www.link.cs.cmu.edu/link/ 
Figure 1 summarizes this architecture.

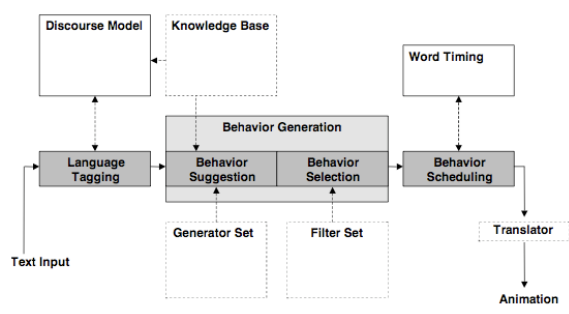

Figure 1. BEAT agent architecture (source: [17])

- Lessons to learn from this work: simplify the animation pipeline by using a three step process: annotate sentences, then relate each behavior using an allinclusive approach then filter out those incoherent ones based on the current context.

- Questions: it is not clear how the knowledge for the characters is obtained and formalized (heuristics are mentioned) into a set of rules.

2) FearNot!: (Fun with Empathic Agents Reaching Novel outcomes in Teaching) [18] describes a simulated environment populated with autonomous virtual characters that aim at promoting an empathic reaction on children playing with characters victim of bullying.

The inspiration for this approach came from theater. A Brazilian Dramaturgist has proposed a role playing enactment exercise for the development of political activism skills. FearNot! then, used an approach where child users were asked to play as an 'invisible friend', advising the victim character, so influencing agent's decisions. The motivation was trying to achieve a sense of responsibility on children while analyzing what just happened.

Characters were implemented based upon an affective agent architecture capable of: perceiving environmental changes (objects, events and other agents) from which emotional state can be appraised. Then, the emotional state can be used (combined with the world state) for the deliberation module in two levels: the schematic level produces impulsive actions, while the coping level considers two approaches (problem-focused and emotion-focused) to produce deliberative actions. All resulting actions are then, executed by the effector of the agent.

- Lessons to learn from this work: A theater metaphor can be gives us a logic, compelling and attractive way of interacting with autonomous self-animated virtual characters.

- Questions: is such a complex agent architecture that involves several layers really necessary? are agents necessary at all?
3) Teatrix: [19], [20] is a game-like interactive learning environment populated with $3 \mathrm{D}$ virtual characters that act autonomously based on specific roles. The project aims at delivering an interactive collaborative environment in which children can create their own stories by choosing scenes and characters and by acting and writing.

In Teatrix, story creation process was divided into three phases: story set-up, story creation and story writing. At story set-up, children can describe the main elements of the story like scenes, characters and items. Scenes are the spatial location for the characters. Characters are specified by their name, type and role (villain, hero, helper, magician, beloved one and beloved relative). Items can be used to extend characters' behaviors (like a magic wand, for instance). In the second phase (story creation), children can select (from a predefined set) all the actions they want the characters to perform. This means that they can create several different stories from a single story set-up (established before). The third phase is the story writing and consists of a recorded movie of the entire usage of the software, allowing the children to store and share their stories, enriching their learning experience.

- Lessons to learn from this work: story set-up, story creation and story writing.

- Questions: instead of simply setting up characters from a predefined set, how difficult it would be to allow them to write their own characters specifying the knowledge involved?

4) Puppet \& CrossTalk: The Puppet project [21] was a system developed to improve learning through improvisational theater. Improvisational theater means to create interesting scenarios for the improvisers to act in, based on a set of explicit or implicit rules like, for instance, the status (the relationships with other characters and with the surrounding environment) and attitude of the characters (a manner of feeling and behaving while pursuing their goals).

CrossTalk [22], [23] is a system that incorporate animated presentation agents in a TV commercial-like situation, in order to present products for sale. It combines two subsystems: cyberella (a conversational agent) and an inhabited marketplace (IMP), which works as a scenario for two virtual actors (Tina and Ritchie). These actors play the roles of a salesman and a customer, respectively, and the IMP works as a virtual showroom with the difference that it is not a mere enumeration of facts about the product nor does it have a fixed discourse for presentation.

It is possible for the user to previously determine: characters' roles, attitudes toward a product, personalities and interests. The IMP sub-module can work in two different modes: presentation (each character plays the role attributed to it) and off-duty (a character will step out of its role, acting as a professional actor), based on a concept called metatheater. Meta-theater creates a mixture of fiction and reality by providing actors with roles and meta-roles, which works 
like if the actor, during the performance, decide to jump out of his role and start 'acting as himself'.

- Lessons to learn from this work: improvisational theater and meta-theater.

- Questions: how improvisational rules are obtained?

5) Virtual Humans: According to Thalmann \& Thalmann [24], a virtual human is "a visualization of the simulation of the behavior of realistic human beings". There are three levels of modeling concerning the development of virtual humans [25]:

1) Realistic appearance modeling refers how to create characters that look like real people. This means studying aspects like hair, clothes and body modeling.

2) Realistic motion modeling refers to ways of representing both path planning and physical locomotion like walking, jumping, etc.

3) Realistic behaviors modeling refers to the creation of behaviors based on models that reflect real human behaviors, most likely inspired by psychology and other human sciences.

For the matter of this work, it is only relevant the study of the behavior models. There are several works that aim at describing how to model, implement and possible applications of virtual humans in terms of behavioral simulation (e.g. [26], [27], [12]) which, in general, lies on psychological models that aim at mimicking real humans in a given context. One common feature that most of these works has described is to use personality traits and emotion models as a way of producing autonomous believable characters capable of showing individuality in such a way that they seem to be motivated by those characteristics just like any human.

- Lessons to learn from this work: Personality traits and emotion models.

- Questions: are such realistic (and complex) simulations of reality really necessary to produce virtual actors? could a simpler (minimal) approach be used instead and still guarantee good degrees of believability?

6) Façade: Mateas and Stern define Façade as "an attempt to create a real-time 3D animated experience akin to being on stage with two live actors who are motivated to make a dramatic situation happen" [28].

In Façade the player acts like a friend trying to intervene in a couple's quarrel. He/she can type sentences that are autonomously interpreted by the characters, changing their 'opinions' and course of actions. The system includes several languages with specific purposes aiming the authoring of the characters and the dramatic situation:

- A Behavior Language allows specification of the behavioral responses for the actors

- Natural Language Understanding is a forwardchaining template language that aims at mapping users typed sentences into discourse acts.
- Reaction Decider describes all possible reactions to discourse acts.

- Beat Sequencing allows management of dramatic situations.

- Lessons to learn from this work: behavior language, language interpretations and reactions via templates and dramatic situations described as beat sequences.

- Questions: how these knowledge templates are described? how scalable is this approach?

7) Master-Servant: In Hayes-Roth et al [29], the authors describe a research on "agents that function as improvisational actors who spontaneously and cooperatively generate their stories at performance time". Their synthetic characters are improvisational actors that are defined by three structures: a plot is a constrained sequence of actions that involves a set of individuals, a role is a class of characters whose prototypical behaviors, relationships, and interactions are known to both actors and audience, and a character is a personality defined as a set of psychological traits.

In order to produce engaging performances, improvisational actors must follow a series of predefined heuristics (directions) like, for instance: accept all offers, not block your partner, do the natural thing, do not try to be clever, reincorporate previously generated elements.

Directing improvisational actors means, in this context, that constrains can be defined for any one of these three elements:

- Role-Constrained Improvisation: establishes which sets of actions are considered 'appropriate' and 'neutral' for each given role. Any actor should respect such constrains and only choose behaviors within these sets. For instance, a servant should open the door for his master.

- Plot-Structured Improvisation: defines particular plot outlines that actors use within their joint performances, like, for instance: in a plot the servant acts 'as usual' and behave submissively and in other plot the servant could defy master's authority by refusing to obey an order.

- Character-Constrained Improvisation: determines the set of behaviors an actor could perform based on a series of variables that stresses the current status of the character. Three variables were suggested by the authors:

1) Demeanor status refers to character's intrinsic behaviors like body posture, jerky movements, hand gestures, and so on.

2) The relationship status refers to character's social behaviors like authority or subordination, avoiding eye contact, pointing fingers, etc.

3) The character's status in space denotes its relationship with the surrounding environment and objects. Is the character willing to interact with 
them or not?

- Lessons to learn from this work: plot, role, character and improvisational directing.

- Questions: how difficult it would be to adapt this model to our definition of virtual actor?

After considering the lessons learned from these works and as an attempt to come up with answers for (some) of the questions raised, a virtual actor metaphor has been proposed. It is presented and discussed in details in the next section.

\section{The Virtual Actor Framework}

As presented before, our understanding is that a virtual actor is an analogy to a real actor, which autonomously, and by its independent interpretation of the situation, can perform its role according to a given script, as part of a story.

After considering the literature review, it is our understanding that an Autonomous Digital Actor (ADA) should be able to perform (at least) the following steps:

1) Script Unit Annotation: an actor should be able to interpret the script from each of its units. A script unit represents one performable action for a character in a story, for instance, a speech or any other actions ${ }^{4}$ that are still susceptible an interpretation. A particular script unit should be annotated with tags (similarly, for instance, to [23]) to allow ADAs to proper interpret it.

2) Acting Performance Suggestion: once a digital actor understands a script unit and what it is expected from it to play, it is time for it decide how to enact it to allow the audience to understand and engage with the story being told.

3) Animation Scheduling: after the virtual actors have decided upon the appropriate actions, it is time for the system to animate them by translating each action into animation commands for an animation engine.

These three steps resemble the architecture proposed by Cassell et al. for BEAT (refer to section II-E1), despite the fact that it significantly differs in terms of the implementation and details.

\section{A. Virtual Actor Architecture}

There are two kinds of professionals involved in the process of creating virtual actors (see Figure 2):

- Acting Teacher: is the person responsible for training virtual actors to perform specific roles. The way to do that is creating the virtual actor's knowledge base starting from the script analysis items that, as presented before in section II-C, represent the knowledge extracted from reading the script to be played.

\footnotetext{
${ }^{4}$ By actions we mean performative actions like walking, open a door, and so on.
}

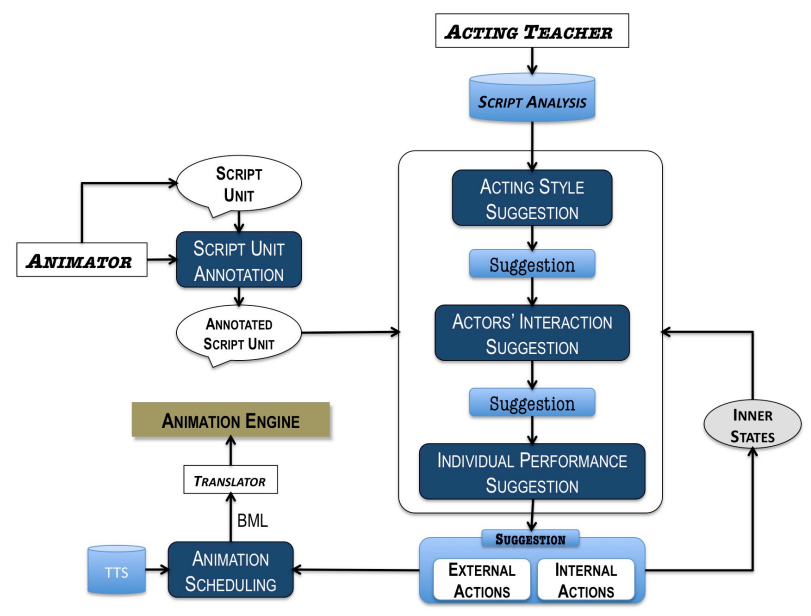

Figure 2. Virtual Actors Proposed Framework

- Animator: is the person responsible for defining the actual script to be performed and annotate each unit in it, so that the virtual actors can suggest a performance.

Our proposed virtual actors framework is composed of five steps:

- Script Unit Annotation: the animator, using this module, is responsible for the writing and annotating of each script unit of the script to be performed. An annotation is composed of three attributes:

1) AgEnt: the responsible for acting the unit.

2) TARGET: to whom the action is addressed.

3) TAGS: a dialog act tag that represents the pragmatics, as for instance "insult", and an appraisal tag that represents eliciting conditions of emotions, for example "good event", of a script unit (cf. [23]).

- Acting Style Suggestion: determine based on the script analysis information what should be the proper acting style for each actor. Examples of acting styles are exaggeration, moderation, optimism, and pessimism.

- Actors' Interaction Suggestion: for each scene, determines coordinated acting approaches. Two possible approaches are collaboration and competition.

- Individual Acting Suggestion: responsible for proposing how each actor should behave while enacting a given script unit. This module takes into consideration the suggestions previously made by the other two modules.

By the end of the suggestion phase, a list of internal and external actions are produced. Internal actions relate to a character's inner states as for example emotional states, hunger or thoughts. External actions represent appropriate physical displays (face, body and voice) of the internal actions. 
- Animation Scheduling: responsible for translating external actions into an animation to later be rendered by a graphics engine. We based our interface on the BML language [30], [31] to specify animations.

An acting suggestion here means the list of actions that should be performed by actors while enacting the script. An acting suggestion is actually a hierarchy of suggestions at different levels of interpretation of the script:

1) the higher level is the script itself. At this level each actor can only decide on the generic acting style to use, based on the theme of the script and some characteristics of its character (refer to section II-C). Examples of acting styles are "exaggerated acting" and "restrained acting".

2) the second level (scene) determines the current situation that the characters are 'living' (a quarreling, a reconciliation, a party, etc.). Knowing this situation helps actors decide on convincing behaviors (those that respect social conventions, laws, culture, etc.).

3) next, at the third level (interaction), the system will provide coordinated acting performances involving the actors of the scene. These interaction suggestion rules should prevent actors from performing contradictory actions.

4) at the level of individual performance, a list of plausible behaviors are suggested that should reflect the actor's intentions when performing a script unit.

5) each individual performance is composed of actions. An action is any instantaneous action that an actor is able to perform like smiling, crying, jumping, etc.

6) describing an action means to combine action units as for instance, raise left brow, move right leg, etc.

\section{CONCLUSIONS}

Virtual actors will represent the next step in authoring 3D movies with believable characters in computer animation. In our understanding, a virtual actor is an analogy to a real actor, which autonomously, and by its independent interpretation of the situation, can perform its role according to a given script, as part of a story.

This article presented an overview of acting theories. We expect that this study contributes to foster the development of a virtual actor framework that incorporates some aspects present in real acting performances, making the process of animating with virtual actors simpler and more intuitive.

This article has suggested an agent architecture that bears similaties to e.g. Cassell's BEAT [17]. The architecture assumes three modules:

- Script Annotation: the animator is responsible by, using a predefined list of tags, suggesting proper interpretations for each unit of the script.

- Behavior Suggestion: composed by three separate 'sub-modules', is responsible for suggesting behaviors at different levels of acting.
- Animation Scheduling: translates each suggestion into a BML-like notation that is later translated into animation commands for an animation engine.

Some of the concepts described in this paper are currently being experimented and implemented as part of the CREACTOR authoring framework[32] (see Figure 3).

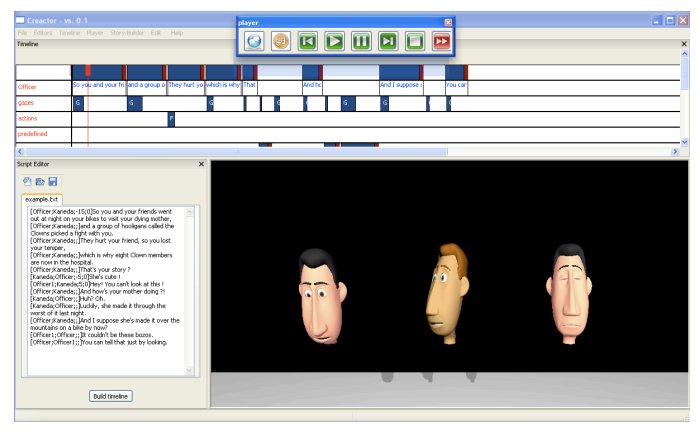

Figure 3. CREACTOR: A Virtual Actors Authoring tool

\section{ACKNOWLEDGEMENTS}

This research is partly funded by the Portuguese Science and Research Foundation FCT (Fundação para a Ciência e a Tecnologia), under reference PTDC/EIA/69236/2006, VirtualActor project.

\section{REFERENCES}

[1] K. Perlin and G. Seidman, "Autonomous digital actors," in Motion in Games, 2008, pp. 246-255. [Online]. Available: http://dx.doi.org/10.1007/978-3-540-89220-5_24

[2] I. A. Iurgel and A. F. Marcos, "Employing personality-rich virtual persons-New tools required," Computers \& Graphics, vol. 31, no. 6, pp. 827-836, Dec. 2007. [Online]. Available: http://www.sciencedirect.com/science? _ob=ArticleURL\&_udi=B6TYG-4PFW6BD-5\&_user= $10 \& \_r d o c=1 \& \_$fmt $=\& \_$orig $=$search $\& \_s o r t=d \& v i e w=c \&$ acct=C000050221\&_version $=1 \&$ \&urlVersion $=0 \&$ _userid= 10\&md5=0a7843da9f168f1bd43471a78a39149e

[3] I. Iurgel, "VirtualActor: endowing virtual characters with a repertoire for acting," in Interactive Storytelling, U. Spierling and N. Szilas, Eds., vol. LNCS 5334. Erfurt, Germany: Springer Verlag, Nov. 2008, pp. 89-91.

[4] C. Stanislavski, An Actor Prepares. Methuen Drama, 1937.

[5] Acting-School-Stop.com, "Acting techniques," http://www.acting-school-stop.com/index.html, Apr. 2010 [Online]. Available: http://www.acting-school-stop.com/ index.html

[6] J. Weston, Directing Actors: Creating Memorable Performances for Film and Television. USA: Michael Wiese Productions, 1996. 
[7] J. Thomas, Script Analysis for Actors, Directors and Designers, 4th ed. Focal Press, 2009.

[8] C. S. Waxberg, The Actor's Script: Script Analysis for Performers, 1st ed. Heinemann Trade, 1998.

[9] J. Cassell, "Nudge nudge wink wink: Elements of Faceto-Face conversation for embodied conversational agents," in Embodied Conversational Agents, J. Cassell, J. Sullivan, S. Prevost, and E. Churchill, Eds. MIT Press, 2000, pp. $1-27$.

[10] G. Ball and J. Breese, "Emotion and personality in a conversational agent," in Embodied Conversational Agents, J. Cassell, J. Sullivan, S. Prevost, and E. Churchill, Eds. MIT Press, 2000, pp. 189-219.

[11] A. Ortony, G. L. Clore, and A. Collins, The Cognitive Structure of Emotions. Cambridge University Press, 1988.

[12] A. Egges, S. Kshirsagar, and N. M. Thalmann, "A model for personality and emotion simulation," in Knowledge-Based Intelligent Information \& Engineering Systems, 2003.

[13] I. Poggi and C. Pelachaud, "Performative facial expressions in animated faces," in Embodied Conversational Agents, J. Cassell, J. Sullivan, S. Prevost, and E. Churchill, Eds. MIT Press, 2000, pp. 155-188.

[14] O. E. Torres, S. Prevost, and J. Cassell, "Turn taking vs. discourse structure: How best to model multimodal conversation," Machine Conversations, pp. 143-154, 1998.

[15] L. Hiyakumoto, S. Prevost, and J. Cassell, "Semantic and discourse information for Text-to-Speech intonation," 1997. [Online]. Available: http://citeseerx.ist.psu.edu/viewdoc/ summary?doi=?doi=10.1.1.26.6312

[16] J. F. Sowa, "Conceptual graphs," in Handbook of Knowledge Representation, F. van Harmelen, V. Lifschitz, and B. Porter, Eds. Elsevier, 2008, pp. 213-237. [Online]. Available: http:// citeseerx.ist.psu.edu/viewdoc/summary?doi=10.1.1.112.6475

[17] J. Cassell, H. H. Vilhjalmsson, and T. Bickmore, "BEAT: the behavior expression animation toolkit," in Proceedings of the 28th annual conference on Computer graphics and interactive techniques. ACM, 2001, pp. 477-486. [Online]. Available: http://portal.acm.org/citation.cfm?id=383315\&dl=

[18] R. S. Aylett, S. Louchart, J. Dias, A. Paiva, and M. Vala, "FearNot! - an experiment in emergent narrative," in Intelligent Virtual Agents. Springer, 2005, pp. 305-316. [Online]. Available: http://dx.doi.org/10.1007/11550617_26

[19] R. Prada, I. Machado, and A. Paiva, "TEATRIX: virtual environment for story creation," in Proceedings of the 5th International Conference on Intelligent Tutoring Systems. Springer-Verlag, 2000, pp. 464-473. [Online]. Available: http://portal.acm.org/citation.cfm?id=745833

[20] A. Paiva, I. Machado, and R. Prada, "Heroes, villains, magicians, ...: dramatis personae in a virtual story creation environment," in Proceedings of the 6th international conference on Intelligent user interfaces. Santa Fe, New Mexico, United States: ACM, 2001, pp. 129-136. [Online]. Available: http://portal.acm.org/citation.cfm?doid= 359784.360314
[21] M. Klesen, "Using theatrical concepts for Role-Plays with educational agents," Applied Artificial Intelligence: An International Journal, vol. 19, no. 3, p. 413, 2005. [Online]. Available: http://www.informaworld.com/10.1080/ 08839510590910237

[22] S. Baldes, P. Gebhard, M. Kipp, M. Klesen, P. Rist, T. Rist, and M. Schmitt, "The interactive CrossTalk installation: MetaTheater with animated presentation agents," in Proceedings of the Workshop on "Lifelike Animated Agents" held in conjunction with the Seventh Pacific Rim International Conference on Artificial Intelligence, 2002, pp. 9-15.

[23] P. Gebhard, M. Klesen, and T. Rist, "Adding the emotional dimension to scripting character dialogues," Proc. of the 4th International Working Conference on Intelligent Virtual Agents (IVA'03), Kloster Irsee, vol. 2003, pp. 48-56, 2003. [Online]. Available: http://citeseerx.ist.psu. edu/viewdoc/summary?doi=10.1.1.89.9891

[24] N. M. Thalmann and D. Thalmann, "The world of virtual actors," Virtual Worlds and Multimedia, John Wiley, pp. 113126, 1993.

[25] N. M. Thalmann and D. Thalmann, "Virtual humans: thirty years of research, what next?" The Visual Computer, vol. 21, no. 12, pp. 997-1015, 2005. [Online]. Available: http://dx.doi.org/10.1007/s00371-005-0363-6

[26] E. de Sevin and D. Thalmann, "A motivational model of action selection for virtual humans," in Computer Graphics International (CGI). New York, NY, USA: IEEE Computer SocietyPress, 2005.

[27] S. Kshirsagar and N. M. Thalmann, "Virtual humans personified," in Proceedings of Autonomous Agents Conference (AAMAS), Jul. 2002, pp. 356-359.

[28] M. Mateas and A. Stern, "Façade, an experiment in building a fully-realized interactive drama," in Game Developers Conference, San Jose, CA., Mar. 2003.

[29] B. Hayes-roth, R. V. Gent, and D. Huber, "Acting in character," in Autonomous Agents and Multi-Agent Systems, 1997, pp. 92-112. [Online]. Available: http: //citeseerx.ist.psu.edu/viewdoc/summary?doi=10.1.1.57.2156

[30] S. Kopp, B. Krenn, S. Marsella, A. Marshall, C. Pelachaud, H. Pirker, K. Thórisson, and H. Vilhjálmsson, "Towards a common framework for multimodal generation: The behavior markup language," in Intelligent Virtual Agents. Springer-Verlag, 2006, vol. 4133, pp. 205-217. [Online]. Available: http://dx.doi.org/10.1007/11821830_17

[31] H. Vilhjálmsson, N. Cantelmo, J. Cassell, N. E. Chafai, M. Kipp, S. Kopp, M. Mancini, S. Marsella, A. Marshall, C. Pelachaud, Z. Ruttkay, K. Thórisson, H. van Welbergen, and R. van der Werf, "The behavior markup language: Recent developments and challenges," in Intelligent Virtual Agents, 2007, pp. 99-111. [Online]. Available: http: //dx.doi.org/10.1007/978-3-540-74997-4_10

[32] I. A. Iurgel, R. E. da Silva, P. R. Ribeiro, A. B. Soares, and M. F. dos Santos, "CREACTOR - an authoring framework for virtual actors," in International Conference on Intelligent Virtual Agents (IVA). Amsterdam, The Netherlands: Springer Verlag, 2009. 